Portland State University

PDXScholar

6-8-2017

\title{
Are There Discrete Symmetries in Relativistic Quantum Mechanics
}

Izzy Pikting Cheung

Portland State University

Follow this and additional works at: https://pdxscholar.library.pdx.edu/honorstheses Let us know how access to this document benefits you.

\section{Recommended Citation}

Cheung, Izzy Pikting, "Are There Discrete Symmetries in Relativistic Quantum Mechanics" (2017).

University Honors Theses. Paper 430.

https://doi.org/10.15760/honors.426

This Thesis is brought to you for free and open access. It has been accepted for inclusion in University Honors Theses by an authorized administrator of PDXScholar. Please contact us if we can make this document more accessible: pdxscholar@pdx.edu. 


\title{
Are There Discrete Symmetries in Relativistic Quantum Mechanics?
}

\author{
by \\ Izzy Pikting Cheung
}

An undergraduate honors thesis submitted in partial fulfillment of the requirements for the degree of

Bachelor of Science

in

University Honors

and

Physics

Thesis Advisor

Prof. Guang-Jiong Ni

Portland State University

2017 


\title{
Are There Discrete Symmetries in Relativistic
}

\section{Quantum Mechanics?}

\author{
Izzy Pikting Cheung ${ }^{1}$ and Guang-jiong $\mathrm{Ni}^{1,2}$ \\ ${ }^{1}$ Department of physics, Portland State University \\ ${ }^{2}$ Fudan University
}

\begin{abstract}
Since the CPT theorem was introduced in the $20^{\text {th }}$ century, it has been one of the most important ongoing projects in particle physics. The CPT invariance helps to indicate if there are discrete symmetries in relativistic quantum mechanics (RQM), the answer to this question is ambiguous. This paper will mainly explain the answers via the timeline and point out misnomers and some missing concepts. We (me and my advisor) claim the answer is there are discrete symmetries in RQM is "yes, but no, no and yes again". The most recent experiment shows the violation of CPT invariance is only $2 \times 10^{-10}$.
\end{abstract}




\section{Introduction}

In 2017, the experiments data shows the violation of CPT invariance is only $2 \times 10^{-10}([1])$, but we also know that the $\mathrm{C}, \mathrm{P}$ and $\mathrm{T}$ individually are violated in weak interactions, but in different degrees. The question is how the combination, CPT can be valid to $100 \%$ ? The answer to the question of Are There Discrete Symmetries in Relativistic Quantum mechanics is "yes, but no, no, and yes again".

The CPT theorem was first introduced by G. Lüders and W. Pauli during 1964-1967 ([2][4]), and it was considered one of the fundamental conservations laws. In 1956, Lee-Yang discovered the $\theta-\tau$ puzzle and started to question the conservation law of parity in weak interactions ([6]). In 1956-1957, Lee-Oehme-Yang theoretically and Wu et al. ([6], [7]) experimentally worked together to figure out, predict and prove the $\mathrm{P}$ and $\mathrm{C}$ are violated for neutrino in weak interactions to nearly $100 \%$. However, the combination of CP was interestingly conserved, because neutrino was considered to be massless at that time. In 1964, Cronin and Fitch et al. ([10]) discovered out CP and in 1970, Schubert discovered T violation in the neutral kaon experiments ([12]). The violation of CP and T showed no discrete symmetry remains valid except CPT invariance in relativistic quantum mechanics (RQM) until 2013. Ni and his collaborators published a paper and claimed ([16]), “(There is) discrete symmetry in RQM.” Ni et al. proved the new symmetry $\mathcal{P} \mathcal{T}=\mathcal{C}$ being equivalent to the CPT invariance, while the original charge conjugation $\mathrm{C}$ is essentially wrong. Furthermore, the hidden antiparticle field in the original EPR experiment for spinless particles ([14]) and the recent new $K^{0} \bar{K}^{0}$ correlation experimental data is analyzed by Ni et al. They emphasize the work by Feshbach and Villars dissociation $(\psi=\varphi+\chi)([17])$ to show KG particle work the same as Dirac particle in RQM. Because of the principle of special relativity, the new symmetry of $\mathcal{P} \mathcal{T}=\mathcal{C}$ immerges as the 
whole universal validity to the foundation of RQM. Lastly, Wigner's time reversal will be discussed in a depth and as why we think it is a misnomer in CPT theorem. Furthermore, CPT invariance guarantee the discrete symmetries in RQM, and $\mathcal{P} \mathcal{T}=\mathcal{C}$ works well for both of Dirac particles and neutrinos as tachyons.

\section{I "Yes": conservation of individual $\mathrm{C}, \mathrm{P}$ and $\mathrm{T}$ symmetry}

\section{C, P and T symmetries before 1955}

In 1954-1957, Lüders and Pauli proved the validity of CPT theorem in quantum field theory (QFT) for the first time ([2]-[4]). The combination of the theoretical proof of CPT theorem and the experimental proof of invariance of CPT was considered as one of the fundamental conservation laws, along with the conservation of energy and conservation of momentum. Here, $\mathrm{C}$ is the charge conjugation and is considered is a discrete symmetry, as $e \rightarrow$ $-e$ and $\psi \rightarrow \psi^{*}$ with $\psi$ is the wavefuntion (WF). Since $\psi$ transforms to $\psi^{*}$, the corresponding energy transforms to "negative" energy $(E \rightarrow-E)$. P is parity and the corresponding conservation law is considered as a discrete symmetry with the transformation of $x \rightarrow-x$. T is the time reversal, and was first introduced by Wigner in 1932. Wigner's time reversal is a transformation on a WF with $\psi(x, t) \rightarrow \psi^{*}(x,-t)$ using equation without considering KleinGordon equation (KG). This can be easily seen from the invariance of Schrödinger equation's WF under time reversal with $t \rightarrow-t$ :

$$
\begin{gathered}
i \hbar \frac{\partial}{\partial t} \psi(x, t)=\widehat{H} \psi(x, t) \\
i \hbar \frac{\partial}{\partial t} \psi^{*}(x,-t)=\widehat{H} \psi^{*}(x,-t)
\end{gathered}
$$

This gives $\psi(x, t) \rightarrow \psi^{*}(x,-t)$. 
Consider the WF of $\mathrm{n}$ terms in a stationary state, the time reversal for this $\mathrm{n}$-terms WF ([4]) is:

$$
\psi_{n}(x, t) \rightarrow \psi_{n}^{*}(x,-t)
$$

with

$$
\begin{gathered}
\psi_{n}(x, t)=u_{n}(x) e^{-\frac{i}{\hbar} E_{n} t} \\
\psi_{n}^{*}(x,-t)=u_{n}^{*}(x) e^{-\frac{i}{\hbar} E_{n} t}
\end{gathered}
$$

Where if $u(x)=e^{\frac{i p \cdot x}{\hbar}}$ and $u^{*}(x)=e^{-\frac{i p \cdot x}{\hbar}}$, and the complex conjugate of $\psi_{n}(x, t)$ in the WF under the Wigner's time reversal is $\psi_{n}^{*}(x,-t)$. So here time reversal is corresponding to the reversal of momentum $p, p \rightarrow-p$, with the energy staying the same. Wigner was clear that the time reversal is actually motion reversal, and he was also right about the use of the energy operator for the particle in Schrödinger equation, because there was no concept of antiparticle at all at that time. But we will see below, the definition of so-called Wigner's time reversal causes a lot of confusion later, and it was not his fault.

\section{Four fundamental interactions}

There are four fundamental interactions in nature that are identified as: weak interaction, strong interaction, electromagnetic interaction and gravitational interaction. Gravitational interaction is also known as gravity, which we experience every day. The electromagnetic interaction is a binding force among electrons and protons in an atom. The strong interaction is the binding force for quarks and forms them together into protons, neutrons or other relatively heavy particles. Quark is one of the elementary particles that have been discovered, and it has 6 "flavors" of up, down, strange, charm, top and bottom. Weak interaction is an interesting force 
that allows quarks to switch among six flavors, this usually happens in the beta-decay $(\beta-$ decay).

\section{II "No": $P$ and $C$ violations and $C P$ conservation}

\section{1. $P$ and $C$ violations}

In 1956-1957, Lee-Yang and Wu et al. ([6]-[9]) worked together, predicted, and then experimentally verified that $\mathrm{P}$ and $\mathrm{C}$ individually violated to nearly $100 \%$ in weak interactions, especially for neutrinos. Because of the $\theta-\tau$ puzzle, Lee-Yang started to question parity conservation in general and especially in weak interactions ([6]). As mentioned in the paper, "Parity nonconservation implies the existence of interactions which mix parities". In other words, if parity were violated, a state of atoms or nuclei would cease to be an eigenstate of a definite parity, but a mixed state of parity and opposite parity. In Lee-Yang's paper, they analyzed carefully the experimental data until 1956 and came to a conclusion that in the electromagnetic and nuclear interactions, the parity conservation law holds in relative high accuracy. The situation in weak interactions including the $\beta-$ decay was not so clear, so there is a great interpretation provided by Lee-Yang ([5], [6]), and they assumed the Hamiltonian diving into two terms,

$$
\widehat{H}=C \widehat{H}_{S}+C^{\prime} \widehat{H}_{P}
$$

where $\widehat{H}_{S}$ and $\widehat{H}_{P}$ are scalar and pseudoscalar with $C$ and $C^{\prime}$ the coupling constants respectively. In the past, the probability of $\beta$-decay to be proportional to the lifetime only, because "...in all of these phenomena no interference terms exist between the parity-conserving and paritynonconserving interactions" ([6]), the probability of $\beta$ - decay is seemingly proportional to $|C|^{2}$ $+\left|C^{\prime}\right|^{2}$ instead of just $|C|^{2}$. Lee-Yang then firmly pointed out the existence of $C^{\prime}$ in eq. (2.1.1), 
and also interprets, “(In eq. (2.1.1)), one has to measure an observable proportional to the interference term $C C^{\prime}$, and such observable must be a pseudoscalar which changes its sign under the space inversion" ([5]). $C \neq 0$ and $C^{\prime} \neq 0$ simultaneously only if $C C^{\prime} \neq 0$.

In this case, use the measurement of angular distribution of electrons coming from $\beta-$ decay of oriented nuclei to determine if the parity is conserved or not, which was suggested in Lee-Yang's 1956 paper [see eq. (2.1.2) and eq. (2.1.3)]. The angular distribution of $\beta-$ decay can be written as

$$
I(\theta) d \theta=(\text { constant })(1+\alpha \cos \theta) \sin \theta d \theta
$$

where $\cos \theta \sim \bar{p} \cdot \bar{S}$ with $\bar{p}$ as its electron's momentum and $\bar{S}$ as nuclei's spin (they are polarized along the $\mathrm{z}$-direction by magnetic field under the low temperature). If $\alpha$ does not equal to 0 , the parity is violated. So $\alpha$ can be written as

$$
\alpha=2\left[\int_{0}^{\pi / 2} I(\theta) d \theta-\int_{\pi / 2}^{\pi} I(\theta) d \theta\right] / \int_{0}^{\pi} I(\theta) d \theta<0
$$

In other words, we can measure emitted electron over $\theta$ between 0 and $90^{\circ}(\pi / 2)$ as an up-half sphere, and $\theta$ between $90^{\circ}$ and $180^{\circ}(\pi)$ as a down-half sphere to determine the angular distribution of $e^{-}$with respect to $\theta$ being up down asymmetric. Wu's experiment verified measurement of the parity violation with a maximal degree.

\section{CP conservation}

Furthermore, Lee-Yang considered combining $\mathrm{P}$ and $\mathrm{C}$ together to check the $\mathrm{CP}$ transformation, and they proved the $\mathrm{CP}$ is $100 \%$ conserved for neutrino ([9]). Since $\mathrm{P}$ and $\mathrm{C}$ are two discrete symmetries, the combined CP is a continuous symmetry. The relationships are shown in Table 1. 


\begin{tabular}{cccc}
\hline & $\mathcal{P}$ & $\mathcal{C}$ & $\mathcal{C P}$ \\
\hline$v_{\boldsymbol{L}}$ & $v_{R}$ & $\bar{v}_{L}$ & $\bar{v}_{R}$ \\
$\overline{\boldsymbol{v}}_{\boldsymbol{R}}$ & $\bar{v}_{L}$ & $v_{R}$ & $v_{L}$ \\
\hline
\end{tabular}

Table 1. $v_{L}$ is the neutrino particle with left helicity, and $\bar{v}_{R}$ is the antineutrino with right helicity. $\mathrm{P}$ is the parity transformation; $\mathrm{C}$ is the charge conjugation transformation. $\mathrm{C}$ and $\mathrm{P}$ violate to $100 \%$ individually in the weak interaction and $\mathrm{CP}$ is the combined transformations together.

Assume $v_{L}$ is the massless neutrino with left helicity, so the corresponding antineutrino should have right helicity as $\bar{v}_{R}$. A massless neutrino has same speed as the speed of light $\mathrm{c}$, and it is longitudinally polarized permanently. This means in this system, only $v_{L}$-particle and $\bar{v}_{R^{-}}$ antiparticle exist, and neither $\bar{v}_{L}$ nor $v_{R}$ exists.

In the table $1, v_{L}$ becomes $v_{R}$ after parity transformation, and $v_{L}$ becomes $\bar{v}_{L}$ after charge conjugation transformation. $\bar{v}_{R}$ becomes $\bar{v}_{L}$ after parity transformation, $\bar{v}_{R}$ becomes $v_{R}$ after charge conjugation transformation. Furthermore, both of $v_{R}$ and $\bar{v}_{L}$ do not exist in nature, but only when $v_{L}$ and $\bar{v}_{R}$ go through both $\mathcal{C P}$ transformations. It seems the violation of $\mathrm{P}$ and $\mathrm{C}$ were recovered by the combination of them, and the story would have a happy ending.

\section{III "No": A neutral $K^{0} \bar{K}^{0}$ experiment and CP violation}

In a neutral $K^{0} \bar{K}^{0}$ system, $K^{0}$ is the neutrally charged particle and $\bar{K}^{0}$ is the neutrally charged antiparticle of $K^{0}$, where 0 is the spin zero. $K^{0}(d \bar{s})$ is composed of a down quark $(d)$ and a strange antiquark $(\bar{s})$, so $\bar{K}^{0}(\bar{d} s)$ is composed of a down antiquark and a strange quark as the antiparticle of $K^{0} . K^{0}$ and $\bar{K}^{0}$ are created in strong interaction which are the eigenstates of the hamiltonian. $K_{1}^{0}$ and $K_{2}^{0}$ are two eigenstates in weak interaction, which are two different linear combination of $K^{0} \bar{K}^{0}$ system with two different main decay modes.

$$
\begin{gathered}
K_{1}^{0} \rightarrow \pi^{+} \pi^{-}, \text {or } \pi^{0} \pi^{0} \\
K_{2}^{0} \rightarrow \pi^{+} \pi^{-} \pi^{0}, \text { or } \pi^{0} \pi^{0} \pi^{0},
\end{gathered}
$$


$K_{1}^{0}$ decays into two final pions whereas $K_{2}^{0}$ decays into three final pions, so CP eigenvalues of $K_{1}^{0}$ is +1 and of $K_{2}^{0}$ is -1 . Let $K_{1}^{0}$ and $K_{2}^{0}$ presented in terms of $K^{0}$ and $\bar{K}^{0}$. Assuming CP is conserved ([11]),

$$
\begin{aligned}
& K_{S}^{0}=K_{1}^{0}=\frac{1}{\sqrt{2}}\left(K^{0}-\bar{K}^{0}\right), \mathrm{CP} K_{1}^{0}=\frac{1}{\sqrt{2}}\left(\bar{K}^{0}+K^{0}\right)=+K_{1}^{0} \\
& K_{L}^{0}=K_{2}^{0}=\frac{1}{\sqrt{2}}\left(K^{0}+\bar{K}^{0}\right), \mathrm{CP} K_{2}^{0}=\frac{1}{\sqrt{2}}\left(-\bar{K}^{0}-K^{0}\right)=-K_{2}^{0}
\end{aligned}
$$

However, the experiment shows the lifetimes of $K_{1}^{0}$ and $K_{2}^{0}$ are different, $K_{1}^{0}$ decays much more quickly than $K_{2}^{0}$. So $K_{1}^{0}$ and $K_{2}^{0}$ are also denoted as $K_{S}$ and $K_{L}$ respectively with different lifetimes $\tau_{S}=0.8923 \times 10^{-8} S, \tau_{L}=5.116 \times 10^{-8} S$, and with mass difference $m_{K_{L}}-m_{K_{S}}=$ $3.484 \times 10^{-12} \mathrm{MeV}$.

In 1964, J. Cronin and V. Fitch's experiment discovered that the $K^{0} \bar{K}^{0}$ system in the weak interaction is with only a little CP violation of $0.3 \%$, because they found $K_{L} \rightarrow \pi^{+} \pi^{-}$, $\pi^{0} \pi^{0}$ too. In 1970, K.R. Schubert et al. ([12]) discovered the time reversal is also violated to $0.3 \%$ in weak interaction in their experiment. Therefore, $\mathrm{C}, \mathrm{P}$, and $\mathrm{T}$ are violated in weak interaction individually, but a combination of CPT is invariant. Hence, in 1965, Lee-Wu proposed the CPT relationship between particle and antiparticle,

$$
|\bar{a}\rangle=\mathrm{CPT}|a\rangle
$$

where $\bar{a}$ is the antiparticle and $a$ is the particle ([13]). The old C (charge conjugation) is no longer the particle and antiparticle transformation. 


\section{IV "Yes": there are discrete symmetries in RQM}

\section{Why Momentum-energy operator for antiparticle was overlooked so long?}

While CPT theorem and CPT invariance are widely accepted, most people still don't accept the momentum- energy operator for antiparticle [eq. (4.1.1)] ([14]) versus the momentumenergy operator for particle [eq. (4.1.2)].

$$
\begin{gathered}
\hat{p}_{c}=i \hbar \nabla, \hat{E}_{c}=-i \hbar \frac{\partial}{\partial t} \\
\hat{p}=-i \hbar \nabla, \hat{E}=i \hbar \frac{\partial}{\partial t}
\end{gathered}
$$

Many people simply just accept one set of momentum-energy equation as eq. (4.1.2), and they might think C-operation can be used to simply transform eq. (4.1.2) to eq. (4.1.1). But C invariance is violated to $100 \%$ in the weak interactions, so we have to accept two sets of momentum-energy equations, which is simply proved by CPT transformation. Experimental data already shows that the antiparticle's energy is always positive, and just like that in particle. But the "hole theory" insists that we could explain the energy of antiparticle is "negative", because the use of the "hole theory" would bring the energy back to positive. Therefore, it is the time to abandon the "hole theory" now.

In 1935, the original Einstein-Podolsky-Rosen (EPR) paper ([14]) discussed an ideal experiment for two spinless particles, which is precisely linked to a $K^{0} \bar{K}^{0}$ correlation experiment in 1998. Both experiments pointed to the necessity of the existence of antiparticle and the opposite momentum-energy operators for antiparticle versus that for particle. The hidden relationship was first pointed out by Ni and his friend Guan (1935-2007) ([15]), and this relationship satisfies four commutation relations:

$$
\left[x_{1}-x_{2}, \hat{p}_{1}+\hat{p}_{2}\right]=0,\left[t_{1}+t_{2}, \hat{E}_{1}-\hat{E}_{2}\right]=0
$$




$$
\left[x_{1}+x_{2}, \hat{p}_{1}-\hat{p}_{2}\right]=0,\left[t_{1}-t_{2}, \hat{E}_{1}+\hat{E}_{2}\right]=0
$$

Here we only consider back-to-back events of $K^{0} \bar{K}^{0}$ correlation, therefore, let $\hat{p}_{1}$ be the momentum of a particle running to the right, and $\hat{p}_{2 c}$ be the momentum of an antiparticle running to the left, $x_{1}=v t_{1}>0, x_{2}=-v t_{2}<0$. Similarly, $\hat{E}_{1}$ is the energy for a particle and $\hat{E}_{2 c}$ is that for an antiparticle. So

$$
\hat{p}=-\hat{p}_{c}, \hat{E}=-\hat{E}_{c}
$$

Eq. (4.1.4) becomes

$$
\left[x_{1}+x_{2}, \hat{p}_{1}+\hat{p}_{2 c}\right]=0,\left[t_{1}-t_{2}, \hat{E}_{1}-\hat{E}_{2 c}\right]=0
$$

There is tiny $\mathrm{CP}$ violation in the coupled equation of $K_{S}^{0}$ and $K_{L}^{0}$, and it can be neglected. As the "strangeness" in this system is an additive quantum number." $K^{0} K^{0}$ or $\bar{K}^{0} \bar{K}^{0}$ are with strangeness but $K^{0} \bar{K}^{0}$ or $\bar{K}^{0} K^{0}$ have zero strangeness. Ni et al. used comparison of intensities of antisymmetric states of system with zero-strangeness and strangeness to test in which state $K^{0} \bar{K}^{0}$ pair was created in their paper in 2013 ([16]).

Both $K^{0}, \bar{K}^{0}$ and $K^{0} \bar{K}^{0}$ pair is created in $J^{P C}=1^{--}$antisymmetric state, so the intensities of zero-strangeness and strangeness are respectively:

$$
\begin{aligned}
& I_{\text {zero }}^{\text {antisym }}\left(t_{a}, t_{b}\right)=\frac{1}{8} e^{-2 \gamma \bar{t}}\left\{e^{-\gamma_{S}\left|t_{a}-t_{b}\right|}+e^{-\gamma_{L}\left|t_{a}-t_{b}\right|}+2 e^{-\gamma\left|t_{a}-t_{b}\right|} \cos \left[\Delta m\left(t_{a}-t_{b}\right)\right]\right\} \\
& I_{\text {strangeness }}^{\text {antisym }}\left(t_{a}, t_{b}\right)=\frac{1}{8} e^{-2 \gamma \bar{t}}\left\{e^{-\gamma_{s}\left|t_{a}-t_{b}\right|}+e^{-\gamma_{L}\left|t_{a}-t_{b}\right|}-2 e^{-\gamma\left|t_{a}-t_{b}\right|} \cos \left[\Delta m\left(t_{a}-t_{b}\right)\right]\right\}
\end{aligned}
$$

where $t_{a}$ and $t_{b}$ are proper times of particle 1 and particle 2 respectively, and $\Delta m=m_{L}-m_{S}$, $\gamma=\frac{1}{2}\left(\gamma_{L}+\gamma_{S}\right), \bar{t}=t_{a}\left(\right.$ for $\left.t_{a}<t_{b}\right)$ and $\bar{t}=t_{b}\left(\right.$ for $\left.t_{a}>t_{b}\right)$. The main difference between eq. (4.1.7) and eq. (4.1.8) is the " + " and " - " in the last terms. The strangeness and zerostrangeness for $K^{0} \bar{K}^{0}$ pair created in antisymmetric state has strongest intensity in EPR limit $\left(t_{a}=t_{b}\right)$ because the strangeness is zero. 
In this EPR limit, $x_{1}+x_{2}=v\left(t_{1}-t_{2}\right)=0, p_{2 c}=-p<0, E_{2 c}=E_{1}>0$, so the $K^{0} \bar{K}^{0}$ system has the lowest eigenvalues $(0,0,0)$ of three commutative operators. These eigenvalues in $K^{0} K^{0}$ events become $0,2 p$ and $2 E$, so they have zero intensity at EPR limit.

\section{FV dissociation of KG equation}

Interestingly, $\mathrm{KG}$ equation was long regarded as less important as Dirac equation in RQM. The new story began in 1958; Feshbach and Villars (FV) ([17]) rethought about how the Klein-Gordon (KG) equation [eq. (4.2.1)] can obtain particle and antiparticle in the system. They divided wavefuntion of $\mathrm{KG}$ equation $\psi$ into two parts as eq. (4.2.2).

$$
\begin{aligned}
\left(i \hbar \frac{\partial}{\partial t}-V\right)^{2} \psi(x, t) & =-c^{2} \hbar \nabla^{2} \psi(x, t)+m^{2} c^{4} \psi(x, t) \\
\psi & =\varphi+\chi
\end{aligned}
$$

Where $\varphi$ represents the particle field and $\chi$ represents the antiparticle field,

$$
\begin{aligned}
\varphi & =\frac{1}{2}\left[\left(1-\frac{V}{m c^{2}}\right) \psi+i \frac{\hbar}{m c^{2}} \dot{\psi}\right] \\
\chi & =\frac{1}{2}\left[\left(1+\frac{V}{m c^{2}}\right) \psi-i \frac{\hbar}{m c^{2}} \dot{\psi}\right]
\end{aligned}
$$

Eq. (4.2.3) and eq. (4.2.4) of FV dissociation obey Schrödinger- like equation and couple together as,

$$
\begin{aligned}
& \left(i \hbar \frac{\partial}{\partial t}-V\right) \varphi=m c^{2} \varphi-\frac{\hbar^{2}}{2 m} \nabla^{2}(\varphi+\chi) \\
& \left(i \hbar \frac{\partial}{\partial t}-V\right) \chi=-m c^{2} \chi+\frac{\hbar^{2}}{2 m} \nabla^{2}(\chi+\varphi)
\end{aligned}
$$

Recall the energy in special relativity (SR) with $\mathrm{V}=0$ :

$$
E= \pm \sqrt{c^{2} p^{2}+m^{2} c^{4}}
$$

therefore, the energy eigenstates of KG equation [eq. (4.2.1)] can be also written as, 


$$
\begin{aligned}
& \psi \sim e^{\left(-\frac{i}{\hbar} E t\right)}, E>0 \\
& \psi \sim e^{\left(-\frac{i}{\hbar} E t\right)}, E<0
\end{aligned}
$$

In this case, we can see energy can be positive or negative, and eq. (4.2.6) can also be written as when $V \neq 0$,

$$
(E-V)^{2}=c^{2} p^{2}+m^{2} c^{4}
$$

and the energy for an antiparticle:

$$
\left(\hat{E}_{c}-V_{c}\right)^{2}=c^{2} \hat{p}_{c}^{2}+m^{2} c^{4}
$$

eq. (4.2.8) satisfies the $\mathrm{KG}$ equation for a particle:

$$
\left(i \hbar \frac{\partial}{\partial t}-V\right)^{2} \psi(x, t)=-c^{2} \hbar \nabla^{2} \psi(x, t)+m^{2} c^{4} \psi(x, t)
$$

and $\mathrm{KG}$ equation for an antiparticle:

$$
\left(i \hbar \frac{\partial}{\partial t}-V\right)^{2} \psi_{c}(x, t)=-c^{2} \nabla^{2} \psi_{c}(x, t)+m^{2} c^{4} \psi_{c}(x, t)
$$

A continuity equation can be derived from eq. (4.2.10) as

$$
\frac{\partial \rho}{\partial t}+\nabla \cdot j=0
$$

where $\rho$ can be expressed as

$$
\rho=\varphi^{*} \varphi-\chi^{*} \chi
$$

There are two differences between Dirac equation and KG equation, 1) As eq. (4.2.5) and eq. (4.2.10) shown, KG equation has the second order of $\frac{\partial}{\partial t}$ while Schrödinger and Dirac equation have first order of $\frac{\partial}{\partial t}$. Schrödinger didn't consider the concept of antiparticles whereas Dirac did. 2) The probability for Schrödinger equation and Dirac equation is always positivedefinite, but it is not for $\mathrm{KG}$ equation. Why for many years, $|\varphi|>|\chi|$, KG particle shows it is a particle whereas $|\varphi|<|\chi|$, the KG particle shows it is an antiparticle. But this is still not good, 
because $\rho$ being a probability density, and it must be positive-definite. This puzzle remains until the Ni et al.'s paper in 2013 ([16]) where FV dissociation for KG particle was further developed into a space-time $(x \rightarrow-x, t \rightarrow-t)$ invariance $\mathcal{P} \mathcal{T}=\mathcal{C}$ as follows,

$$
\begin{gathered}
\left\{\begin{array}{c}
x \rightarrow-x, t \rightarrow-t \\
V(\boldsymbol{x}, t) \rightarrow-V(\boldsymbol{x}, t)=V_{c}(\boldsymbol{x}, t)
\end{array}\right. \\
\left\{\begin{array}{l}
\psi(\boldsymbol{x}, t) \rightarrow \mathcal{P} \mathcal{T} \psi(\boldsymbol{x}, t)=\psi_{c}(\boldsymbol{x}, t) \\
\varphi(\boldsymbol{x}, t) \rightarrow \mathcal{P} \mathcal{T} \varphi(\boldsymbol{x}, t)=\chi_{c}(\boldsymbol{x}, t) \\
\chi(\boldsymbol{x}, t) \rightarrow \mathcal{P} \mathcal{T} \chi(\boldsymbol{x}, t)=\varphi_{c}(\boldsymbol{x}, t)
\end{array}\right.
\end{gathered}
$$

Apply $\mathcal{P} \mathcal{T}=\mathcal{C}$, eq. (4.2.14) to eq.(4.2.13):

$$
\begin{aligned}
\rho & \rightarrow \mathcal{P} \mathcal{T} \rho=\rho_{c} \\
& =\chi_{c}^{*} \chi_{c}-\varphi_{c}^{*} \varphi_{c}
\end{aligned}
$$

and suppose $\rho_{c}$ could be positive or zero if $\rho$ is negative, and puzzle for decades is finally solved.

In retrospect, Feshbach and Villars's historical contribution to physics and even to natural science and sociology. One of their major contributions is that they unveiled an universal principle of nature: nothing is pure in this world. Everything is impure because there are always two sides of confrontation inside. There is no exception to elementary particles: $\psi=\varphi+\chi, \varphi$ means a hidden particle field, and $\chi$ is a hidden antiparticle field. The realistic KG particle shows up as a particle or an antiparticle depending on which of $\varphi$ field or $\chi_{c}$ field is in charge inside $\left(|\varphi|>|\chi|\right.$ or $\left.\left|\chi_{c}\right|>\left|\varphi_{c}\right|\right)$.

\section{$3 \mathcal{P} \mathcal{T}=\mathcal{C}$ as the essence of special relativity (SR)}

The principle of the SR and the nonrelativistic quantum mechanic (NRQM) are two ingredients that keep RQM (KG equation and Dirac equation) work. The principle of SR is much deeper than general relativity. The latter seems more complicated and difficult to learn, but it is 
essentially a classical field theory. General relativity doesn't consider the relationship of particle and antiparticle, but only the gravity and curvature of spacetime. It is well known that SR is characterized by the invariance of the Lorentz transformation, and which links infinite inertial frames moving each other with relative arbitrary speed $v(v / c<1)$ along the same direction. There is one invariant with respect to infinite continuous transformation (without $v$ explicitly) being,

$$
\begin{aligned}
& c^{2}\left(t_{1}-t_{2}\right)^{2}-\left(\boldsymbol{x}_{1}-\boldsymbol{x}_{2}\right)^{2} \\
= & c^{2}\left(t_{1}^{\prime}-t^{\prime}{ }_{2}\right)^{2}-\left(\boldsymbol{x}_{1}^{\prime}-\boldsymbol{x}_{2}^{\prime}\right)^{2} \\
= & \mathrm{constant}
\end{aligned}
$$

Now we have another discrete symmetry $\mathcal{P} \mathcal{T}=\mathcal{C}(x \rightarrow-x, t \rightarrow-t)$ which can keep the equation eq. (4.3.1) invariant. In order to show $\mathcal{P} \mathcal{T}=\mathcal{C}$ is of dynamical nature transforming a particle to its antiparticle. Let's write down another two invariances for a particle and an antiparticle respectively, and consider the particle and antiparticle into Lorentz transformation in terms of energy and momentum. Eq. (4.3.2) and eq. (4.3.3) show, particle and antiparticle are equal under the mass inversion.

$$
\begin{aligned}
& E^{2}-c^{2} \boldsymbol{p}^{2}=E^{\prime 2}-c^{2} \boldsymbol{p}^{\prime 2}=m^{2} c^{4} \\
& E_{c}{ }^{2}-c^{2} \boldsymbol{p}_{c}{ }^{2}={E^{\prime}}_{c}{ }^{2}-c^{2}{\boldsymbol{p}^{\prime}}_{c}{ }^{2}=m_{c}{ }^{2} c^{4}=m^{2} c^{4}
\end{aligned}
$$

where eq. (4.3.2) and eq. (4.3.3) are proved by our mass inversion $(m \rightarrow-m)$ as eq. (4.3.4).

$$
\begin{gathered}
\left\{\begin{aligned}
m & \rightarrow-m_{c}=-m \\
V(\boldsymbol{x}, t) & \rightarrow V(\boldsymbol{x}, t)=V_{c}(\boldsymbol{x}, t)
\end{aligned}\right. \\
\left\{\begin{array}{l}
\psi(\boldsymbol{x}, t) \rightarrow \psi_{c}(\boldsymbol{x}, t) \\
\varphi(\boldsymbol{x}, t) \rightarrow \chi_{c}(\boldsymbol{x}, t) \\
\chi(\boldsymbol{x}, t) \rightarrow \varphi_{c}(\boldsymbol{x}, t)
\end{array}\right.
\end{gathered}
$$


when $m \rightarrow-m$, there is $\widehat{\boldsymbol{p}} \rightarrow-\widehat{\boldsymbol{p}}_{c}$, and $\widehat{E} \rightarrow-\widehat{E}_{c}, i . e .-i \hbar \nabla \rightarrow-i \hbar \nabla, i \hbar \frac{\partial}{\partial t} \rightarrow i \hbar \frac{\partial}{\partial t}$. In short, for a classical theory being relativistic or not, the simplest criterion is to see whether it is invariant or not under the mass inversion $(m \rightarrow-m)$.

Hence, we see the new discrete symmetry (in one inertial frame like the room I am standing at rest). It is easy to use the continuous Lorentz transformation involving infinite inertial frames. On the contrary, we can say the thousands of experiments, which support for the validity of SR as well as for $\mathcal{P} \mathcal{T}=\mathcal{C}$ symmetry.

\section{Why use strong reflection and hermitian conjugation to prove CPT theorem?}

In this section, we try to present the proof of CPT theorem, which is simply but rigorous notation. We propose that the strong reflection proposed by Pauli is expressed as $\widehat{\mathcal{P T}}$, and which is the counterpart of $\mathcal{P} \mathcal{T}=\mathcal{C}$ (at the level of RQM) at the level of QFT. In the Fock space, the C, $\mathrm{P}$ and T transformations at the level of RQM can be expressed as $\hat{C}, \widehat{P}$ and $\widehat{T}$ at the level of QFT respectively.

In the following, begin from Lee-Wu's particle-antiparticle CPT symmetry as eq. (4.3.4), eq. (4.3.5) shows the new transformation of $\mathcal{P} \mathcal{T}=\mathcal{C}$ symmetry for particle $\left|a_{\boldsymbol{p}, h}\right\rangle$ and antiparticle $\left|\bar{a}_{\boldsymbol{p},-h}\right\rangle$ is after the $\hat{C} \hat{P} \widehat{T}$ transformation in eq (4.3.6). The strong reflection was explained by Pauli in ref. [4], "When the space-time coordinates change their sign, every particle transforms into its antiparticle simultaneously." Now, suppose the particle $\left|a_{\boldsymbol{p}, h}\right\rangle=\hat{a}_{\boldsymbol{p}, h}^{\dagger}|0\rangle$ with a definite helicity, and use strong reflection and hermitian conjugation to prove this relationship.

$$
|\bar{a}\rangle=\hat{C} \hat{P} \widehat{T}|a\rangle
$$




$$
\begin{gathered}
\left\{\begin{array}{c}
\hat{C}\left|a_{p, h}\right\rangle=\left|\bar{a}_{p, h}\right\rangle \\
\hat{P}\left|a_{p, h}\right\rangle=\left|a_{-p,-h}\right\rangle \\
\widehat{T}\left|a_{p, h}\right\rangle=\left|a_{-p, h}\right\rangle
\end{array}\right. \\
\hat{C} \hat{P} \hat{T}\left|a_{p, h}\right\rangle=\hat{C} \hat{P}\left|a_{-p, h}\right\rangle=\hat{C}\left|a_{p,-h}\right\rangle=\left|\bar{a}_{p,-h}\right\rangle
\end{gathered}
$$

On the other hand, the Pauli's strong reflection means eq.(4.3.7),

$$
\widehat{\mathcal{P \mathcal { T }}} \hat{a}^{\dagger}{ }_{\boldsymbol{p}, h} \widehat{\mathcal{P T}}^{-1}=\hat{b}_{\boldsymbol{p},-h}
$$

Use the rule (which was missed in Ni's paper) as eq. (4.3.8),

$$
\hat{A} \cdots \rightarrow \widehat{B} \widehat{\mathcal{P T}}|0\rangle=\langle 0| \hat{A} \cdots \widehat{B}
$$

we can derive the following (4.3.9)-(4.3.10).

$$
\begin{aligned}
\widehat{\mathcal{P T}}\left|a_{\boldsymbol{p}, h}\right\rangle & =\widehat{\mathcal{P T}} \hat{a}^{\dagger}{ }_{\boldsymbol{p}, h} \widehat{\mathcal{P T}}^{-1} \widehat{\mathcal{P \mathcal { T }}}|0\rangle \\
& =\hat{b}_{\boldsymbol{p},-h} \widehat{\mathcal{P T}}|0\rangle=\langle 0| \hat{b}_{\boldsymbol{p},-h}
\end{aligned}
$$

take hermitian conjugation of the (4.3.9)

$$
\begin{gathered}
{\left[\widehat{\mathcal{P T}}\left|a_{\boldsymbol{p}, h}\right\rangle\right]^{\dagger}=\left[\langle 0| \hat{b}_{\boldsymbol{p},-}\right]^{\dagger}=b^{\dagger}{ }_{\boldsymbol{p},-}|0\rangle} \\
\left|a_{\boldsymbol{p}, h}\right\rangle \stackrel{\begin{array}{c}
\text { Hermitian conjugion }+ \\
\text { Hertion }
\end{array}}{\longrightarrow}\left|\bar{a}_{\boldsymbol{p},-h}\right\rangle
\end{gathered}
$$

which coincides with the (4.3.6). After the detailed transformations of eq. (4.3.9) and (4.3.10), we eventually have eq (4.3.11) and that proves strong reflection plus hermitian conjugation can successfully prove the CPT theorem in Fock space. Q.E.D

\section{Summary}


There are some modifications on basic concepts in physics discussed as follow:

\section{Is Wigner's time reversal a continuous symmetry or a discrete symmetry?}

Wigner's time reversal involves both of $t \rightarrow-t$ and $\psi(x, t) \rightarrow \psi^{*}(x,-t)$. This is also explained in detail in these two excellent books of J. J. Sakurai ([18], [19]), where Sakurai pointed out that name of Wigner's "time reversal" is just a misnomer. It would be much better to be renamed as the "motion reversal". Unfortunately, Sakurai's advice was overlooked for so long, even after the discovery of Kobayashi and Maskawa's successful explanations on the tiny violation T-symmetry. This misnomer was pointed out again after the publication of Ni et al.'s paper ([16]) in 2013 .

Wigner was clear his “time reversal” is a continuous symmetry. Since Wigner's time reversal is so complicated at the level of RQM with spin, many people including me might be confused about whether the meaning of time reversal is a discrete symmetry or not? After the discovery of T violation, Kobayashi and Maskawa successfully described the tiny CP or T violation into a small phase angle in the Cabbibo- Kobayashi-Maskawa (CKM) matrix of unitary transformation ([20], [21]) between eigenstates of quarks in strong interactions and that in weak interactions within the standard model of particle physics. We highly appreciate Kobayashi and Maskawa's contribution to the particle physics and provided this mechanism. They emphasize again $\mathrm{CP}$ or $\mathrm{T}$ is actually a continuous symmetry. Because unlike a continuous symmetry, we believes the discrete symmetry either is conserved to $100 \%$ or is violated to $100 \%$, like the parity symmetry.

The question is if $\mathrm{T}$ is a continuous symmetry, then CPT is a continuous symmetry as well. How can it be equivalent to our $\mathcal{P} \mathcal{T}=\mathcal{C}$, which is a discrete symmetry obviously? The answer is the old $\mathrm{C}$ survived in the CPT transformation because the two complex conjugations 
canceled out with each other in $\mathrm{C}$ and $\mathrm{T}$ transformations fortunately. $t \rightarrow-t$ in $\mathrm{T}$ and $x \rightarrow-x$ in $\mathrm{P}$ (these two are discrete symmetries combined into one discrete symmetry, a new $\mathcal{P} \mathcal{T}=\mathcal{C}$ ), as long as we insist $\mathrm{C}$ being a transformation of particle to antiparticle without the change the signs of $x$ and $t$. So CPT invariance is equivalent to the symmetry of $\mathcal{P} \mathcal{T}=\mathcal{C}$ exactly. Hence, we can still use the old $\mathrm{C}$ in further research work, but we should never forget that the definition of $\mathrm{C}$ or $\mathrm{T}$ is essentially wrong or a misnomer? Therefore, both $\mathrm{C}$ and $\mathrm{T}$ cannot be valid to $100 \%$.

\section{There are discrete symmetries in relativistic quantum mechanics (RQM)}

\section{1 Symmetries in $\mathcal{P} \mathcal{T}=\mathcal{C}$ for Dirac particles and neutrino as tachyons}

We have three discrete symmetries in RQM, in which one is a universal invariance and two are partially valid (either $100 \%$ correct or $100 \%$ wrong).

\begin{tabular}{lccc}
\hline & $\mathcal{P}=\boldsymbol{P}$ & $\mathcal{T}$ & $\mathcal{P} \mathcal{T}=\boldsymbol{C}$ \\
& $(\boldsymbol{x} \rightarrow-\boldsymbol{x})$ & $(\boldsymbol{t} \rightarrow-\boldsymbol{t})$ & \\
\hline Dirac particle & $\sqrt{ }$ & $\times$ & $\sqrt{ }$ \\
$\boldsymbol{v}, \overline{\boldsymbol{v}}$ as tachyons & $(100 \%)$ & $(100 \%)$ & $(100 \%)$ \\
& $\times$ & $\sqrt{ }$ & $\sqrt{ }$ \\
Types of & $(100 \%)$ & $(100 \%)$ & $(100 \%)$ \\
symmetry & Discrete & Discrete & Discrete \\
\hline
\end{tabular}

Table 2. Three individual transformations in $\mathcal{P T}=\mathcal{C}$ transformation between Dirac particle and tachyons. $\mathcal{P}$ is the parity transformation and $\mathcal{T}$ is the time inversion while keeping $\mathcal{P} \mathcal{T}=\mathcal{C}$ invariance.

In table 2 , there are only $t$ and $x$ with their inversions, but never including $e \rightarrow-e$. We emphasize the time inversion $\mathcal{T}(t \rightarrow-t)$ as the counterpart of $\mathcal{P}$. Interestingly, the Dirac particle has parity conservation but violates the $\mathcal{T}$ inversion to $100 \%$. On the contrary, neutrinos as tachyons $(v, \bar{v})$ violate the parity conservation to $100 \%$ but keep the $\mathcal{T}$ inversion valid to $100 \%$. Similarly, a discrete symmetry can only either be $100 \%$ valid or $100 \%$ violated. In 2017 , the experiment shows "CPT invariance is consistent at a relative precision about $2 \times 10^{-10}$ " ([1]). 
The value of $2 \times 10^{-10}$ indicates the violation of the universal symmetry $(\mathrm{CPT}=\mathcal{P} \mathcal{T}=\mathcal{C})$ in all interactions doesn't exceed $2 \times 10^{-10}$.

Furthermore, there are two things are implied in table 2. 1) Individual space or time inversion is not universally correct. This reveals the space and time are essentially tightly related, so inversion of either of them cannot be totally incorrect. This is why the product of a "no" and a "yes" gives a "yes", and there are obviously three discrete symmetries under three transformations $(\mathcal{P}, \mathcal{T}$ and $\mathcal{P} \mathcal{T}=\mathcal{C})$. 2) $\mathcal{P} \mathcal{T}=\mathcal{C}$ reveals a new symmetry, and it implies the combined spacetime inversion is equivalent to the transformation of particles and antiparticles. Clearly enough, the definition of inversion between particles and antiparticles is just residing in this symmetry transformation $(\mathcal{P T}=\mathcal{C})$, and not comes from elsewhere. This is just what a specific natural law in physics can do. Just like Newton's second law defines mass in $F=m a$, and mass $(m)$ is just residing in this equation, and not coming from elsewhere.

\section{2 Symmetries in $\mathrm{C}, \mathrm{P}, \mathrm{CP}, \mathrm{T}$, and CPT}

\begin{tabular}{|c|c|c|c|c|c|}
\hline & $\begin{array}{c}\mathrm{C} \\
\left(\psi \rightarrow \psi^{*}\right)\end{array}$ & $\begin{array}{c}\mathbf{P} \\
(x \rightarrow-x)\end{array}$ & $\begin{array}{c}\mathbf{C P} \\
\left(\psi \rightarrow \psi^{*}\right) \\
(x \rightarrow-x)\end{array}$ & $\begin{array}{c}\mathbf{T} \\
\left(\psi \rightarrow \psi^{*}\right) \\
(t \rightarrow-t)\end{array}$ & CPT \\
\hline $\begin{array}{l}\text { Dirac } \\
\text { particle } \\
(v \leq c)\end{array}$ & $\begin{array}{c}\text { Yes } \\
\text { (But no for } \\
\text { neutrino, } m=0 \text { ) }\end{array}$ & $\begin{array}{l}\text { Yes } \\
\text { (But no for } \\
\text { neutrino, } m=0 \text { ) }\end{array}$ & $\begin{array}{l}\text { Yes } \\
\text { (But no for } K^{0} \bar{K}^{0} \text { to a } \\
\text { tiny degree) }\end{array}$ & $\begin{array}{c}\text { Yes } \\
\text { (But no for } K^{0} \bar{K}^{0} \\
\text { to a tiny degree) }\end{array}$ & $\begin{array}{c}\text { Yes } \\
(100 \%)\end{array}$ \\
\hline $\begin{array}{l}\text { Neutrino } \\
\text { as } \\
\text { tachyons } \\
(v>c)\end{array}$ & $\begin{array}{c}\text { No } \\
(100 \%)\end{array}$ & $\begin{array}{c}\text { No } \\
(100 \%)\end{array}$ & $\begin{array}{l}\text { Yes } \\
(100 \%) \\
\text { (See discussion } \\
\text { below) }\end{array}$ & $\begin{array}{l}\text { Yes } \\
\quad(100 \%) \\
\text { (See discussion } \\
\text { below) }\end{array}$ & $\begin{array}{c}\text { Yes } \\
(100 \%)\end{array}$ \\
\hline $\begin{array}{l}\text { Type of } \\
\text { symmetry }\end{array}$ & Discrete & Discrete & Continuous & Continuous & Continuous \\
\hline \multicolumn{6}{|c|}{$\begin{array}{l}\text { Table 3. Five individual transformations in CPT transformations between Dirac particle and tachyons. } C \text { is the charge conjugation } \\
\text { transformation, } P \text { is parity transformation, } T \text { is the time reversal and } C P \text { is a combination of } C \text { and } P \text { transformations. }\end{array}$} \\
\hline \multicolumn{6}{|c|}{ For the massive Dirac particle with $v<c$ in free motion, the $\mathrm{CP}\left(\psi \rightarrow \psi^{*}, x \rightarrow-x\right)$} \\
\hline
\end{tabular}


symmetries. Hence, the fact of $\mathrm{C}, \mathrm{P}$ and $\mathrm{T}$ is violated individually to different degrees in different case, and this fact means that none of them is essentially correct. Especially, $\mathrm{C}$ is actually wrong from the beginning, because in which the particle and antiparticle are detached from the spacetime. T is just a misnomer as a "time reversal", because it is not a basic time inversion $(t \rightarrow$ $-t$ only). However, $\mathrm{CPT}=\mathcal{C}=\mathcal{P} \mathcal{T}$ shows up as a continuous symmetry in table 3 , and unlike $\mathrm{C}$ is a discrete symmetry. Therefore, we think that is a reflection of the definition of $\mathrm{C}$ and $\mathrm{T}$ being essentially incorrect, and the requirement of SR dictates the space and time should be inverted simultaneously. Therefore, the inversions of space and time work as one discrete symmetry not separately.

\section{3 Why consider neutrinos as tachyons in two above tables?}

Neutrinos are interesting because they violate the parity to $100 \%$ and only $v_{L}, \bar{v}_{R}$ exist whereas $v_{R}, \bar{v}_{L}$ are forbidden, even though they do have "mass". We believe the best candidates for neutrinos are just tachyons. If we consider relative velocity (between two coordinate systems) exceeds some critical values, we stay in one of these two systems, and will see the tachyon particle looks like running backward in time (or a violation of causality), or energy of particle changes from positive to negative. This strange result is also called tachyons paradox (see Appendix 1). In order to find the solution of this paradox, we have to admit the existence of antineutrinos as antitachyons and use its momentum-energy operators. This explanation of tachyon paradox is similar to the $\mathcal{T}$ symmetry for tachyons in table 2 . In some sense, the existence of tachyonic neutrinos builds a bridge between the old notation CPT and the new notation $\mathcal{C}=\mathcal{P} \mathcal{T}$. 


\section{4 The "arrow of time"}

Look back in table 2, it seems we overlooked $\mathcal{T}$-violation $(t \rightarrow-t)$ of Dirac particle for a long time. Dirac particle's $\mathcal{T}$-violation has a huge impact, because it implies the existence of “arrow of time" at a microscopic level. In 1876, Loschmidt's paradox pointed out that there is "arrow of time" implied by the second law of thermodynamics at a macroscopic level. But

Newton's second law $F=m a=m \frac{d^{2} r}{d t^{2}}$ shows a particle's motion could reverse at a microscopic level, and this is why people definite the time reversal in WF in QM accordingly. Now, it seems the $\mathcal{T}$-violation of Dirac particle does show the "arrow of time" at a microscopic level. So, we should also admit the "arrow of time" is at both microscopic and macroscopic levels, and it's time to happily end the solution of the Loschmidt's paradox.

We are made of Dirac particles, and that is why we can feel "arrow of time" inside our bodies to adapt the evolution of surrounding environment. We must admit the principle of causality before we can recognize the object world. Hence, time $(t)$ is not a coordinate of particle, rather it is an invention of human being. It is always moving forward from past to present and to the future, and never turning back. Accordingly, use one set of energy operator we are able to distinguish the particle $(E>0)$ from the antiparticle $(E<0)$, because of the existence of "arrow of time". Furthermore, we use two sets of energy operators so that antiparticle has the positive energy as $E_{c}>0$. By contrast, the space orientation of $x$ is arbitrary. So momentum of the particles or antiparticles can be positive or negative without any limitations. 


\subsection{A proposal}

After so many events happened since1956, it's time for the contemporary theoretical particle physics to correct the momentum-energy operator in RQM from one set to two sets. At least in teaching RQM, we should let our young generations to learn two sets of momentumenergy operators, let them to make their own judgment. 


\section{Appendix 1. (Appendix 9B in [2])}

In 2000 , there are two experiments showing superluminal propagation of either microwave [50] or a light pulse [51]. Whatever they look amazing, physicists believe the laws of physics (especially, theory of SR) remain intact (e. g. [52]). However, as stressed by some authors, the experimental data about neutrino mass-square

strongly hint that the neutrino might be a superluminal particle [53].

1. An explanation on possible negative mass-square of neutrinos

The square of neutrino mass is measured in tritium beta decay experiments $\left({ }^{3} \mathrm{H}\right.$ $\left.\rightarrow{ }^{3} \mathrm{He}+e^{-}+\bar{\nu}_{e}\right)$ to be negative as reported at Particle Table in 1996 and 2000 [54], where it reads :

$$
m^{2}\left(\nu_{c}\right)=-2.5 \pm 3.3 \mathrm{eV}^{2} .
$$

The pion decay experiments $\left(\pi^{+} \rightarrow \mu^{+}+\nu_{c}\right)$ also show a similar puzzle that [54]:

$$
m^{2}\left(\nu_{\mu}\right)=-0.016 \pm 0.023 \mathrm{MeV}^{2} .
$$

These data, though far from accurate, yet strongly suggest the following kinematic relation for neutrinos:

$$
E^{2}=c^{2} p^{2}-m_{s}^{2} c^{4},
$$

where $E, p$ and $m$, are energy, momentum and "proper mass" of the neutrino respectively e. g. , $m_{s}\left(\nu_{e}\right)=1.6 \mathrm{eV}$ according to (9B. 1) $\left(m^{2}=-m_{s}^{2}\right)$.

Based on (9B. 3) with quantum relations $E=\hbar \omega$ and $p=\hbar k$, the velocity of particle $u$ should be identified with the group velocity $u_{g}=\frac{d \omega}{d k}$ of wave versus the phase velocity $u_{p}=\frac{\omega}{k}$. Defining the changeable (total) mass $\tilde{m}$ by $p=\tilde{m} u_{g}=\tilde{m} u$, one can easily prove that:

$$
p=\tilde{m} u=\frac{u_{p} u_{g}=c^{2},}{\sqrt{\frac{u_{s}^{2}}{c^{2}}-1}}, E=\tilde{m} c^{2}=\frac{m_{s} c^{2}}{\sqrt{\frac{u^{2}}{c^{2}}-1}}
$$

Although Eqs. (9B. 5) are known for many years to describe the superluminal motion $(u>c)$ of a particle (also called the "tachyon" in literature), they do need a quantum derivation and interpretation. To derive (9B. 3) and following Dirac's idea as discussed in $\S 4.1 \mathrm{~B}$, Chang found a Dirac-type equation as follows [55]:

$$
\left\{\begin{array}{l}
i \hbar \frac{\partial}{\partial} \psi_{s}=i c \hbar \alpha \nabla \psi_{s}+\beta_{s} m_{s} c^{2} \psi_{s} \\
\psi_{s}=\left(\begin{array}{l}
\varphi \\
\chi
\end{array}\right), \alpha_{i}=\left(\begin{array}{cc}
0 & \sigma_{i} \\
\sigma_{i} & 0
\end{array}\right), \beta_{s}=\left(\begin{array}{cc}
0 & I \\
-I & 0
\end{array}\right)
\end{array}\right.
$$

A plane-wave solution of it yields (9B. 3) immediately. However, in comparison with Eq. (9.1.17), now $\beta_{s}$ is not a hermitian matrix since $\beta_{s} \neq \beta_{s}^{\dagger}\left(\beta_{s}^{2}=-1\right)$. Usually the violation of hermitian property will lead to instability of solutions. What does it mean in the present situation and how could it be valid?

2. Equation for superluminal neutrino versus Dirac equation $[56,58,60,61]$

As discussed in $\S 3.8 \mathrm{C}$, since the discovery of parity violation in 1956 , the theory for neutrinos is based on the Weyl equation:

$$
i \hbar \frac{\partial}{\partial t} \xi=i c \hbar \sigma \cdot \nabla \xi
$$


where $\boldsymbol{\xi}(\boldsymbol{x}, t)$ is a two-component spinor function. Eq. (9B. 7) describes a positive $\because$ energy $(E>0)$ solution for the left-handed neutrino (with helicity $\lambda=\frac{1}{2}\langle\sigma \cdot \hat{\boldsymbol{p}}\rangle=-$ $\left.\frac{1}{2}, \hat{\boldsymbol{p}}=\frac{\boldsymbol{p}}{|\boldsymbol{p}|}\right)$ and a negative energy $(E<0)$ solution for the right-handed antineutrino (with helicity $\lambda=\frac{1}{2}$ ) in accordance with that verified by experiments. The alternative possibility that:

$$
i \hbar \frac{\partial}{\partial} \eta=-i c \hbar \sigma \cdot \nabla \eta
$$

was thus abandoned. As now experiments show that the neutrino mass is not zero, we assume a new equation for a neutrino being composed of both $\xi$ and $\eta$ coupling via a nonzero $m_{s}$ (or "rest mass" $m_{0}$ to derive Dirac equation with subscript $D$ for comparison).

$$
\begin{aligned}
i \hbar \frac{\partial}{\partial} \xi_{D}=i c \hbar \sigma \cdot \nabla \xi_{D}+m_{0} c^{2} \eta_{D}, \\
\hbar \frac{\partial}{\partial} \eta_{D}=-i c \hbar \sigma \cdot \nabla \eta_{D}+m_{0} c^{2} \xi_{D}, \\
\text { (9B. 9) }
\end{aligned} \mid\left\{\begin{array}{c}
i \hbar \frac{\partial}{\partial t} \xi=i c \hbar \sigma \cdot \nabla \xi-m_{s} c^{2} \eta, \\
\hbar \frac{\partial}{\partial} \eta=-i c \hbar \sigma \cdot \nabla \eta+m_{s} c^{2} \xi,
\end{array}\right.
$$

$\varphi_{D}=\frac{1}{\sqrt{2}}\left(\xi_{D}+\eta_{D}\right), \chi_{D}=\frac{1}{\sqrt{2}}\left(\xi_{D}-\eta_{D}\right)$

$$
\begin{aligned}
& \varphi=\frac{1}{\sqrt{2}}(\xi+\eta), \chi=\frac{1}{\sqrt{2}}(\xi-\eta), \\
& i \hbar \frac{\partial}{\partial} \varphi=i c \hbar \sigma \cdot \nabla \chi+m_{s} c^{2} \chi, \\
& \hbar \frac{\partial}{\partial} \chi=i c \hbar \sigma \cdot \nabla \varphi-m_{s} c^{2} \varphi .
\end{aligned}
$$$$
i \hbar \frac{\partial}{\partial} \varphi_{D}=i c \hbar \sigma \cdot \nabla \chi_{D}+m_{0} c^{2} \varphi_{D} \text {, }
$$$$
\hbar \frac{\partial}{\partial} \chi_{D}=i c \hbar \sigma \cdot \nabla \varphi_{D}-m_{0} c^{2} \chi_{D} .
$$

This is Dirac Eq. (9.1.19) up to a sign change in the $\alpha$ matrix. The continuity equation reads :

$$
\begin{aligned}
& \frac{\partial}{\partial t} \rho_{D}+\nabla \cdot j_{D}=0, \\
& \rho_{D}=\varphi_{D}^{\dagger} \varphi_{D}+\chi_{D}^{\dagger} \chi_{D}=\xi_{D}^{\dagger} \xi_{D}+\eta_{D}^{\dagger} \eta_{D}, \\
& j_{D}=-c\left(\varphi_{D}^{\dagger} \sigma \chi_{D}+\chi_{D}^{\dagger} \sigma \varphi_{D}\right) \\
& \quad=-c\left(\xi b \sigma \xi-\eta_{D}^{\dagger} \sigma \eta_{D}\right) .
\end{aligned}
$$$$
\frac{\partial \rho}{\partial t}+\nabla \cdot j=0,
$$

This is Eq. (9B. 6) for the neutrino.

$$
\mid \begin{gathered}
\rho=\varphi^{\dagger} \chi+\chi^{\dagger} \varphi=\xi^{\dagger} \xi-\eta^{\dagger} \eta, \\
j=-c\left(\varphi^{\dagger} \sigma \varphi+\chi^{\dagger} \sigma \chi\right) \\
=-c\left(\xi^{\dagger} \sigma \xi+\eta^{\dagger} \sigma \eta\right) .
\end{gathered}
$$


First, we see that for the same form of continuity equation (9B. 12), the probability density $\rho$ and probability current density $j$ for a neutrino are different from that for a Dirac particle. The normalization condition $\int \rho d x=1$ (or -1 ) for (9B. 13) corresponds to conservation of helicity in motion: $\lambda=-\frac{1}{2}$ for a neutrino $\nu_{L}$ (or $\lambda=\frac{1}{2}$ for a antineutrino $\bar{\nu}_{R}$ ). Their permanently longitudinal polarization features can be maintained in any inertial frame because of their velocities $u>c$.

Next, we can find a radical difference between (9B. 9) D and (9B. 9). Under the space inversion $(\boldsymbol{x} \rightarrow-\boldsymbol{x})$ and related transformation:

$$
\xi(-x, t) \rightarrow \eta(x, t), \quad \eta(-x, t) \rightarrow \xi(x, t)
$$

Dirac Eq. (9B. 9) D is invariant whereas (9B. 9) fails to do so because of the opposite sign in mass term. It is just a clearcut reflection of the fact that a neutrino yields the maximum violation of parity. The new observation is the parity violation being triggered by its nonzero proper mass (no matter how tiny it is) which in turn implies that the neutrino must be a superluminal particle with permanent helicity — while $\nu_{L}$ and $\bar{\nu}_{R}$ are allowed, $\nu_{R}$ and $\bar{\nu}_{L}$ must be forbidden strictly.

Third, the more important observation lies in the common essence that both (9.1. 19) and (9B. 11) are invariant under the space-time inversion with transformation (9. 5. 16) which does exhibit itself as a basic symmetry. By contrast, the space-inversion symmetry (9B. 15) is a smaller one and can be violated in the case of (9B. 9). Therefore, the violation of hermitian property in Eq. (9B. 6) is stemming from that of parity and thus not displayed via the instability of four solutions for a same momentum but is realized by two of them $\left(\nu_{R}\right.$ and $\left.\bar{\nu}_{L}\right)$ being forbidden strictly with other two solutions $\left(\nu_{L}\right.$ and $\left.\bar{\nu}_{R}\right)$ stabilized. (Ex. 9.9).

3. Two parameters describing a free electron or superluminal neutrino

We are now in a position to realize the marvelous kinematical feature of a superluminal particle together with that of a subluminal one. Consider a plane-wave solution $\xi \sim \eta \sim \exp [i(p x-E t) / \hbar]$ along $x$ axis for a free particle with helicity $\lambda=-$ $\frac{1}{2}$, we have:

$$
\eta=\frac{m_{s} c^{2}}{c p+E} \xi \cdot(|\xi / \eta|>1)
$$

from (9B. 9) and

$$
\chi=\frac{c p-m_{s} c^{2}}{E} \varphi,(|\varphi / \chi|>1)
$$

from (9B. 11). As in $\S 9.5 \mathrm{C}$, we now define generally a "impurity ratio" $R$ for the "hidden amplitude of antiparticle field" to that of "particle field" in a particle being:

$$
R \equiv \sqrt{\frac{\chi^{\dagger} \chi}{\Phi^{\dagger} \varphi}}=\left[\frac{\frac{u}{c}-\sqrt{\frac{u^{2}}{c^{2}}-1}}{\frac{u}{c}+\sqrt{\frac{u^{2}}{c^{2}}-1}}\right]^{\frac{1}{2}},(u>c)
$$




$$
R=\left[\frac{1-\sqrt{1-\frac{u^{2}}{c^{2}}}}{1+\sqrt{1-\frac{u^{2}}{c^{2}}}}\right]^{1 / 2},(u<c)
$$

Similarly, we define a "Weyl parameter" $W$ as the ratio of "hidden amplitude of right-handed helicity field" to that of "left-handed helicity field" in a particle with helicity $\lambda=-\frac{1}{2}$ explicitly :

$$
\begin{gathered}
W=\sqrt{\frac{\eta^{\dagger} \eta}{\xi^{\dagger} \xi}}=\sqrt{\frac{u-c}{u+c}},(u>c) \\
W=\sqrt{\frac{c-u}{c+u}},(u<c) .
\end{gathered}
$$

Being functions of $\frac{u}{c}$, both values of $R$ and $W$ are symmetric with respect to $\frac{u}{c}=1$ in the logarithmic scale. So we define a "rapidity" $\zeta$ of particle with speed $u$ in the whole range $(0, \infty):^{*}$

$$
\zeta=\ln \sqrt{\left|\frac{c+u}{c-u}\right|},(0<u<\infty)
$$

and find that both $R$ and $W$ can be expressed in a unified manner:

$$
R=\tanh \left(\frac{\zeta}{2}\right), \quad W=e^{-\zeta}
$$

which in turn are anticorrelated each other also in a unified way:

$$
R=\frac{1-W}{1+W}, \quad W=\frac{1-R}{1+R}
$$

The parameter $R$ could be understood as a measure of "impurity" of a particle being a superposition state of two hidden contradictory fields $\varphi$ and $\chi\left(\left|\frac{\varphi}{\chi}\right|>1\right)$. Although superficially, a free electron (neutrino) is always a particle with lepton number $L=1$ , it does change intrinsically with its velocity. The larger $R$ is, the larger mass (energy) and more instability the particle will have.

Similarly, the Weyl parameter is a measure of "intrinsic instability of helicity" for a particle with superficial helicity $\lambda=-\frac{1}{2},\left(\left|\frac{\xi}{\eta}\right|>1\right)$. While an electron can turn its helicity to $\lambda=\frac{1}{2}$ when $\left|\frac{\eta}{\xi}\right|>1$, a neutrino's helicity is linked to its lepton number $L$ definitely $\left(L=1, \lambda=-\frac{1}{2}\right.$ whereas $L=-1, \lambda=\frac{1}{2}$ ). The common anticorrelation between $R$ and $W$ for both subluminal and superluminal particles implies that a high energy particle being more "impure" $(R \rightarrow 1)$ will be more stable in helicity $(W \rightarrow 0)$. On the contrary, a particle being unstable in helicity $(W \rightarrow 1)$

- For the case $u>c, \zeta$ is actually a measure of "energeticity" rather than "rapidity". 
corresponds to a "relatively pure" particle $(R \rightarrow 0)$ with low energy. A prominent difference between a Dirac particle and neutrino is that for the former $\lim _{u \rightarrow 0} E=m_{0} c^{2}$ whereas for the latter $\lim _{u \rightarrow \infty} E=0$. The latter case could be understood by (9B. 13) where the normalization condition poses no constraint on $\xi$ and $\eta$ separately, so the limiting behavior of $\lim _{u \rightarrow \infty} W=1$ implies that both $\xi$ and $\eta$ increase infinitely and cancel each other at $u \rightarrow \infty$.

Therefore, the finite and changeable mass of a fermion is not stemming from one kind of excitation which varies continuously inside but some manifestation of coherent cancellation effect between two fields rotating with opposite helicities implicitly, either one of them ${ }^{*}$ can be excited infinitely in essence.

We have to be cautious to talk about "hidden $\chi$ (or $\eta$ ) field". 'For example, if a neutrino has $W=\frac{1}{3}$, we cannot say that "it is composed of $75 \%$ (or $90 \%$ ) lefthanded rotating state and $25 \%$ (of $10 \%$ ) right-handed rotating state" because it is in $100 \%$ left-handed helicity state explicitly while both $\xi$ and $\eta$ field increase drastically inside. Only in an antineutrino with $\left|\frac{\eta_{c}}{\xi_{c}}\right|>1$, can $\eta_{c}$ display itself as a right-handed rotating state, so does $\xi_{c}$ follow accordingly.

Therefore, we should not interprete the "hidden probability amplitude" too materialized in ordinary Language. All fantastic behaviors of particle are due to the linear superposition and interference effect of fields between $\varphi$ and $\chi$ (or $\xi$ and $\eta$ ), not due to their intensities ( $\varphi^{\dagger} \varphi$ etc). The existence of superluminal particle and its marvelous feature are new manifestation of subtlety of QM. In some sense, a particle is also a "Schrödinger's cat" but in microscopic scale and could be compared to a recent experiment discussed at the end of Appendix 10B.

4. Superluminal paradox and neutrino [57], [61].

Consider two inertial frames $\sum$ and $\sum^{\prime}$ moving with relative velocity $v(-c<v$ $\langle c$ ) along $x$ axis. Then the Lorentz transformation (LT) reads :

$$
x^{\prime}=\frac{x-v t}{\sqrt{1-\frac{v^{2}}{c^{2}}}}, \quad t^{\prime}=\frac{t-\frac{v x}{c^{2}}}{\sqrt{1-\frac{v^{2}}{c^{2}}}}
$$

where $(x, t)$ and $\left(x^{\prime}, t^{\prime}\right)$ are space-time coordinates of a same particle $P$ in $\sum$ and $\sum$ frames respectively.

If $P$ is a subluminal particle with uniform velocity $u<c$, there is no any problem. see Fig 9B. 1(a) and (b).

However, for a superluminal particle $P$ with $u>c$, a strange phenomenon occurs. While Fig 9B. 2(a) seems not so different from that for $u<c$ case, Fig. 9B. 2

- They could be viewed as two fundamental vortex excitations of "ether" (i. e. , “yuanqi" or "primary gas " see $\S 10.3 \mathrm{E}$ and Fig 10.3.2) which reminds us of the fact that only two quantized values of angular-momentum for electron spin can be measured along one direction. 

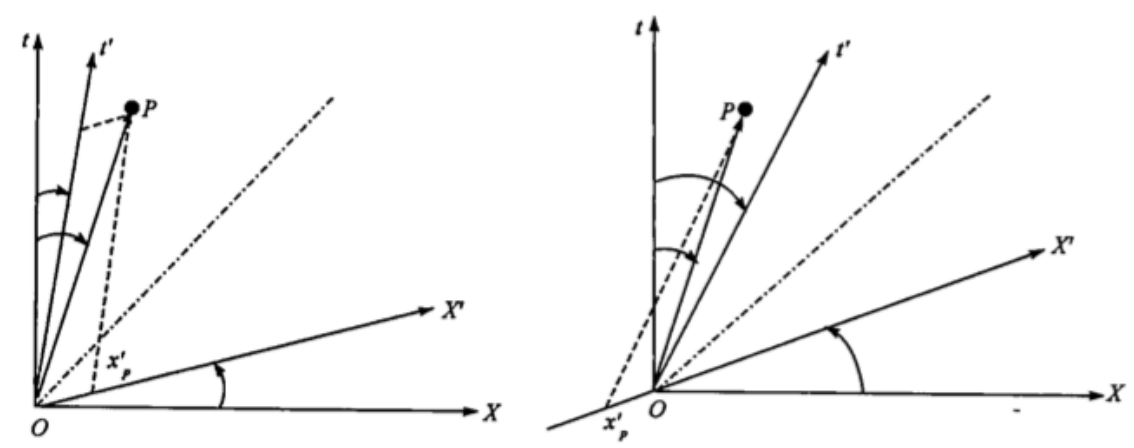

Fig. 9B. 1 A subluminal particle (P) moving along $x$ axis with velocity $u<c$.

(a) $v<u$; (b) $v>u$. ( $v$ is the velocity of $\sum^{\prime}$ frame relative to $\sum$. In the limit $v \rightarrow c, x^{\prime}$ and $t^{\prime}$ axes coincide at the diagonal dash-dot line).

(b) shows a great surprise that when $v>\frac{c^{2}}{u}, t_{P}^{\prime}$ becomes negative:

$$
t^{\prime}{ }_{P}<0, \quad\left(u>c, v>\frac{c^{2}}{u}\right)
$$

which was regarded as the "tachyon traveling backwared in time" or "violation of causality" and has been remaining in literature as a mysterious puzzle (see. e. g. [59]).

In our opinion. The above puzzle can be better displayed in an alternative way. From (9B. 24), one has the addition law for velocity in $L T$ as :

$$
u^{\prime}=\frac{u-v}{1-\frac{u v}{c^{2}}},
$$

( $u=\frac{d x}{d t}, u^{\prime}=\frac{d x^{\prime}}{d t^{\prime}}$ ). Notice that though it is an analytic function of three variables $u$, $u^{\prime}$ and $v$ as long as $|v|<c$ and $u<c$, it does have a singularity if $u>c$. The pole is located at $v=\frac{c^{2}}{u}$ or $u=\frac{c^{2}}{v}$ as shown in Fig 9B. 3.

When $v$ increases across the singularity $\frac{c^{2}}{u}, u^{\prime}$ will leap abruptly from $+\infty \rightarrow-\infty$;

$$
u^{\prime}<(-c),\left(u>\frac{c^{2}}{v} \text { or } v>\frac{c^{2}}{u}\right)
$$

Half of above phenomenon can also be seen in Fig 9B. 2(b) when we gradually rotate the $x^{\prime}$ axis approaching the $O P$ line counterclockwise : $u^{\prime}=\frac{\Delta x^{\prime}}{\Delta t^{\prime}} \rightarrow \infty$. But it seems to us that Fig. 9B. 2(b) is meaningless, the $x^{\prime}$ axis is not allowed to leap across the $O P$ line. We prefer to accept (9B. 27) rather than $t^{\prime}{ }_{p}<0$.

However, Eq. (9B. 27) still remains as a puzzle. To see it, we study the momentum $p^{\prime}$ and energy $E^{\prime}$ of a superluminal particle in the $\Sigma^{\prime}$ frame by $L T$ : 

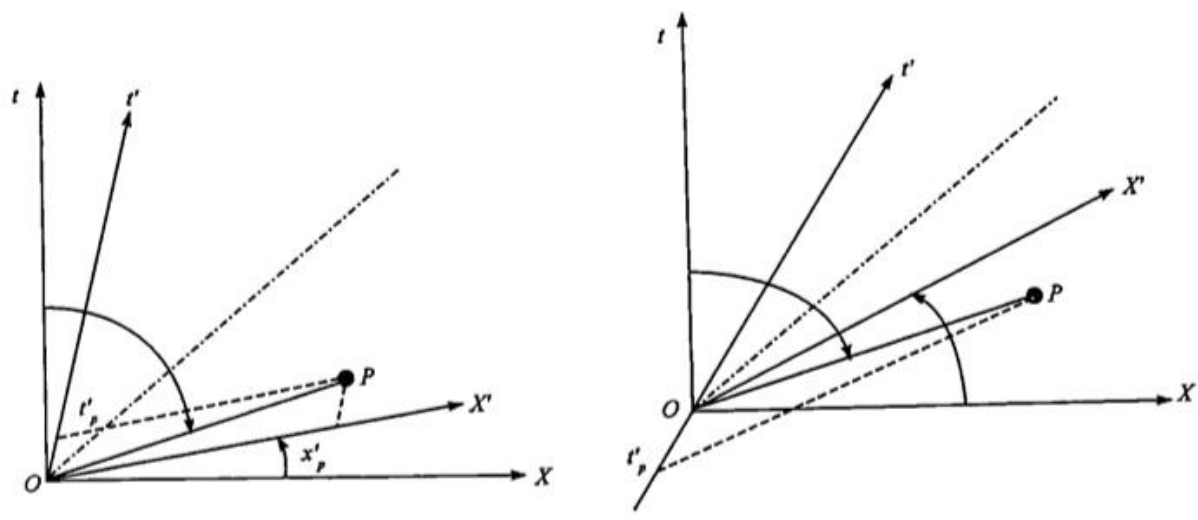

Fig. 9B. 2 A superluminal particle (P) moving along $x$ axis with velocity $u>c$.

$$
\begin{aligned}
& \text { (a) } v<\frac{c^{2}}{u}, t^{\prime},>0, \text { (b) } v>\frac{c^{2}}{u}, t^{\prime},<0 . \\
& p^{\prime}=\frac{p-\frac{v E}{c^{2}}}{\sqrt{1-\frac{v^{2}}{c^{2}}}}, \quad E^{\prime}=\frac{E-v p}{\sqrt{1-\frac{v^{2}}{c^{2}}}}
\end{aligned}
$$

with

$$
p=\frac{m_{s} u}{\sqrt{\frac{u^{2}}{c^{2}}-1}}>0, \quad E=\frac{m_{s} c^{2}}{\sqrt{\frac{u^{2}}{c^{2}}-1}}>0
$$

in $\sum$ frame. Combination of them leads to:

$$
\begin{gathered}
p^{\prime}=\frac{m_{s}}{\sqrt{1-\frac{v^{2}}{c^{2}}} \sqrt{\frac{u^{2}}{c^{2}}-1}}(u-v)>m_{s} c>0, \\
E^{\prime}=\frac{m_{s}}{\sqrt{1-\frac{v^{2}}{c^{2}}} \sqrt{\frac{u^{2}}{c^{2}}-1}}\left(c^{2}-u v\right)<0,\left(u>\frac{c^{2}}{v}, \text { or } v>\frac{c^{2}}{u}\right) .
\end{gathered}
$$

Now the puzzle arises: how can a particle have $u^{\prime}<0\left(u>\frac{c^{2}}{v}\right)$ whereas its $p^{\prime}>0$ ? Moreover, how can its energy become negative in $\sum^{\prime}$ frame: $E^{\prime}<0$ ? (Both $p^{\prime}$ and $E^{\prime}$ vary smoothly at $u v=c^{2}$ ) What do they mean? All above puzzles: (9B. 25), (9B. 27), (9B. 29) and (9B. 30) comprise the "superluminal paradox".

Some authors regarded the paradox as a signal showing that the theory of $L T$. might not be valid for a superluminal particle. We don't think so. We believe the paradox being stemming from the classical nature of above discussion. Once we find:a reasonable quantum theory, the paradox will disappear.

Actually, Let's assume the particle being a neutrino and obeying the equation 

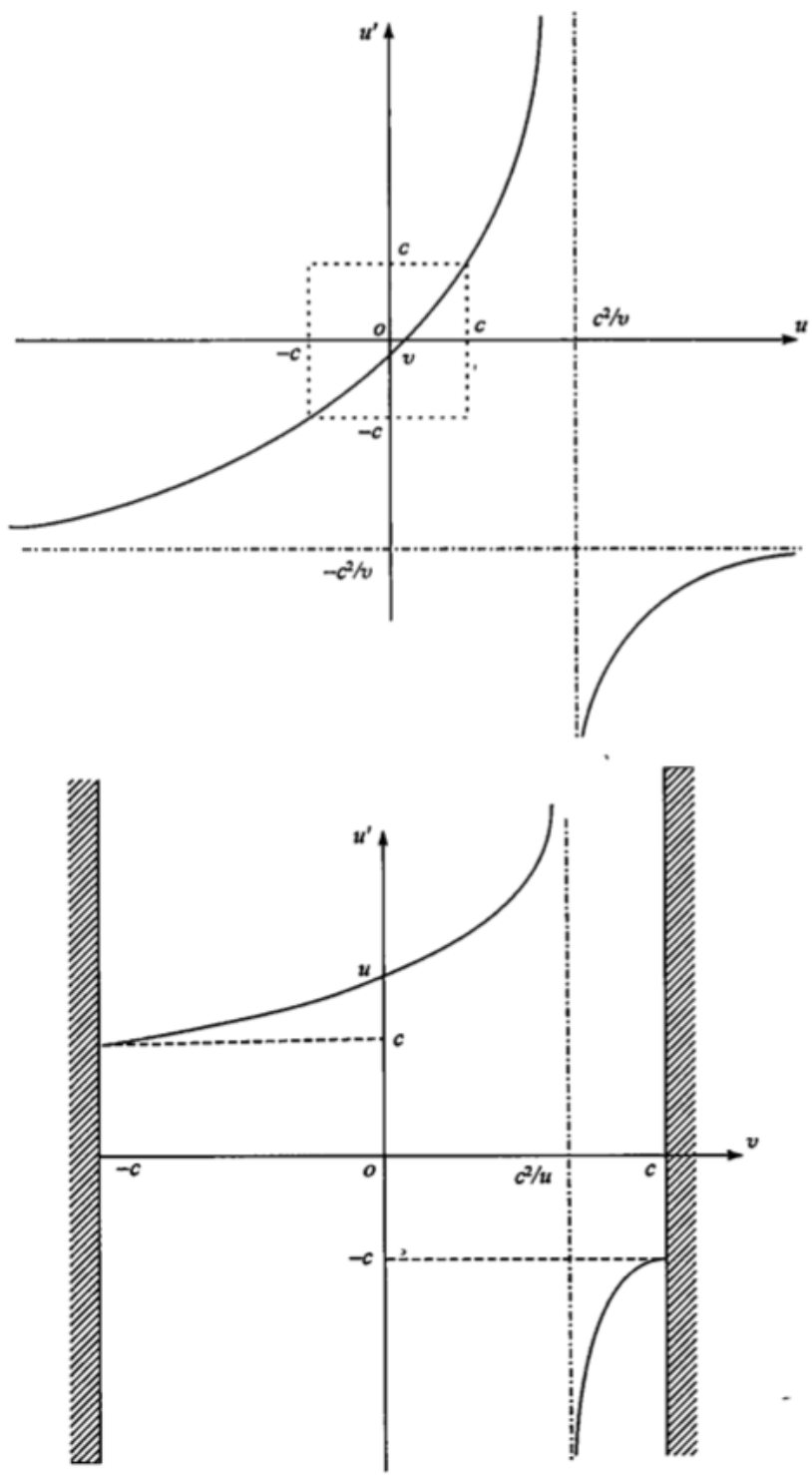

Fig. 9B. 3 Addition of velocity in Lorentz transformation

(a) $u^{\prime}$ as a function of $u$ for a fixed $v$;

(b) $u^{\prime}$ as a function of $v$ for a fixed $u\left(>_{c}\right)$.

discussed in previous subsection and prove that an observer in $\Sigma^{\prime}$ frame will see the neutrino with $u>\frac{u^{2}}{v}$ in $\sum$ frame as an antineutrino since $E^{\prime}<0$. And its momentum is $p^{\prime}{ }_{c}=-p<0$ (instead of $p^{\prime}>0$ ) just in conformity with its velocity $v^{\prime}<0$.

[Proof]. Eq. (9B. 9) is invariant under the following "pure time inversion": 


$$
t \rightarrow-t, \xi(x,-t) \rightarrow \eta_{c}(x, t), \eta(x,-t) \rightarrow \xi_{c}(x, t)
$$

with a concrete solution for particle:

$$
\xi \sim \eta \sim \exp \left[\frac{i}{\hbar}(p x-E t)\right],\left(\left|\frac{\xi}{\eta}\right|>1\right)
$$

being transformed into an antiparticle:

$$
\eta_{c} \sim \xi_{c} \sim \exp \left[\frac{i}{h}(p x+E t)\right] \sim \exp \left[-\frac{i}{h}\left(p_{c}-E_{c} t\right)\right],\left(\left|\frac{\eta_{c}}{\xi_{c}}\right|>1\right)
$$

with $p_{c}=-p<0, E_{c}=E>0$, (where operators for an antiparticle, Eq. (9.4.36), have been used). Q. E. D. Now it is clear that the mysterious time-reversal, (9B. 25 ), is nothing more than a false appearance of the sign change in the phase of wave function, which, of course, can not be reflected suitably in Fig 9B. 2(b). So now all puzzles disappear. There is no paradox at all.

The implication of Fig 9B. 3 is amazing. If we tentagively identify the $\sum$ frame with the rest frame of cosmos in which the $3 \mathrm{~K}$ microwave radiation background is strictly isotropic, our earth ( $\sum^{\prime}$ frame) is moving with velocity $v=365 \mathrm{~km} / \mathrm{s}$. Then the originally isotropic neutrinos (identified tentatively with the dark matter) with velocity distribution in the range $(-\infty<\mathrm{u}<-\mathrm{c}, \mathrm{c}<\mathrm{u}<\infty)$ will be divided anisotropically in the $\sum$ ' frame into two parts. One of them (with $u>\frac{c^{2}}{v}$ ) will be transformed into antineutrinos moving in the opposite direction of $v\left[u^{\prime}<\left(-\frac{c^{2}}{v}\right)\right.$, see Fig 9B. 3(a)]. As an ideal experiment, if we wish to chase a superluminal neutrino with fixed $u$ by increasing our velocity $v$ (Fig 9B. 3(b)), its behavior looks fantastic. First it flees away with speed $u^{\prime}$ even faster and faster $\left(u^{\prime}>u\right)$ until $u^{\prime}=\infty$ when $v \rightarrow$ $\frac{c^{2}}{u}$. Then if we further accelerate to pass the critical value $v_{c}=\frac{c^{2}}{u}$, it changes suddenly into an antineutrino moving toward us $\left(u^{\prime}<-c\right)$. (This is why the $x^{\prime}$ axis can not leap across the OP line in Fig 9B. 2(b)). On the other hand, if we leave the neutrino along opposite direction $(v<0)$, its velocity $\left(u^{\prime}>0\right)$ slows down instead.

5. Summary and discussion:

(a) Numerous experimental tests have been supporting the validity of SR, which stands even more firm than ever before. However, based on the new experimental fact about neutrino, it is possible to construct a superluminal theory compatible with SR.

(b) In particular, the LT (Eqs (9B. 24)) and (9B. 28) and the addition law for velocity (Eq. (9B. 26)) are valid for both subluminal and superluminal phenomena as long as $|v|<c$. This is because the concept about space-time is formed by observers ourselves who are composed of ordinary particles. Our discussion can be meaningful. only if it is based on SR, LT and the invariance of speed of light.

(c) The superluminal paxadox is over. All puzzles stemming from the classicaI', concepts disappear in a reasonable quantum theory. Indeed, the superluminal problem: poses a very severe and interesting test on the validity of Eq. (9B. 9) i. e. , (9B. 11), which in turn is based on the new concept about the symmetry between particle and 
antiparticle as well as on the parity, violation property of neutrinos.

(d) At first sight, the existence of a rest frame $\Sigma=\Sigma_{0}$ in cosmos (implied by the $3 \mathrm{~K}$ microwave radiation background) leads to the violation of the symmetry in LT: other inertial frame $\Sigma^{\prime}$ with velocity $v \neq 0$ relative to $\Sigma_{0}$ is not equavalent to $\Sigma_{0}$. But if $\Sigma_{0}$ is selected by the neutrino (dark matter) (its velocity distribution being isotropic in $\Sigma_{0}$ ), the equal existence of antineutrino with mutual transformation between them as shown in Fig 9B. 3 does imply that the Lorentz symmetry is restored implicitly. In other words, the Lorentz symmetry is hidden in superluminal neutrino bath, it is actually extended to totality of inertial frames with velocity in the whole range $(-c<v<c)$ and realized by neutrino and antineutrino together. 


\section{References}

[1] M.Ahmadi at al., Nature 241 506-512 (2017)

[2] G. Lüders, Kgl. Danske Vidensk. Selsk. Mat-Fys. Medd. 28 (1954)

[3] G. Lüders, Annals of Phys (New York), 2, 1-15 (1957)

[4] W. Pauli, Exclusion Principle, Lorentz Group and Reflection of Space-Time and Charge, W.Pauli et al. Niels Bohr and the Development oh Physics, McGraw-Hill, NewYork, 30-51 (1955)

[5] G. J. Ni and S. Chen, Advanced Quantum Mechanics, Rinton Press (2002)

[6] T. D. Lee and C.N.Yang, Phys Rev., 104, 254-258 (1956)

[7] T. D. Lee, R. Oehme and C.N.Yang, ibid, 105, 340-345 (1957)

[8] C.S. Wu et al., Phys Rev., 105 1413-1415 (1957)

[9] T. D. Lee and C.N.Yang, ibid, 105, 1671-1675 (1957)

[10] J.H.Christensen, J.W. Cronin, V.L.Fitch and R. Turlay, Phys Rev. Lett. 13, 138-140 (1964)

[11] O. Nachtmann, Elementary Particle Physics concepts and phenomena, Springer-Verlag 26 438-449 (1990)

[12] K.R. Schubert et al. Phys Lett. B, 31, 662-665 (1970)

[13] T.D. Lee and C.S. Wu, Annu Rev Nucl Sci. 15, 381-476 (1965)

[14] A. Einstein, B. Podolsky and N. Rosen, Phy Rev., 47, 777-780 (1935) doi:10.1103/PhysRev.47.777.

[15] G. J. Ni, H. Guan, W. M, Zhou and J. Yan, Chin Phy Rev., 17, 393-395 (2000) doi: 10.1088/0256-308X/17/6/002.

[16] G.J. Ni, S. Chen and J. Xu, J Mod Phys, 4 651-675 (2013) www.scirp.org/journal/jmp

[17] H. Feshbach and F.Villars, Rev Mod Phys., 30, 24-45 (1958)

doi:10.1103/RevModPhys.30.24 
[18] J. J. Sakurai, Advanced Quantum Mechanics, Addison-Wesley Publishing Company, Boson (1978)

[19] J. J. Sakurai, Modern Quantum Mechanics, John Wiley \& Sons, Inc., NewYork (1964).

[20] N. Cabbibo, Phys Rev Lett., 10 (12), 531-533 (1963) doi:10.1103/PhyRevLett.10.531

[21] M.Kobayashi and T. Maskawa, Prog Theor Phys 49(2), 652-657 (1973)

doi:10.1143/PTP.49.652 